\title{
SOBRE A CONVERGÊNCIA DAS CADEIAS DE MARKOV
}

\author{
$\begin{array}{lll}\text { F. S. BATISTA } & \text { C. C. SANTOS } \quad \text { L. ANDRADE } \\ & 1\end{array}$ \\ J. C. ARAÚJO R. G. MÁRQUEZ ${ }^{2}$
}

\begin{abstract}
Resumo
As cadeias de Markov são representadas por matrizes quadradas $P_{i j}$ de ordem $r$ e indicam a probabilidade de transição do estado $e_{j}$ para o estado $e_{i}$. O objetivo deste artigo é provar o teorema da convergência de matrizes estocásticas regulares usando tão somente o produto matricial e conhecimentos elementares de análise real, em particular o uso de desigualdades na reta. Uma aplicação prática é apresentada a luz dos três métodos utilizados nesse estudo para a obtenção do vetor estacionário.
\end{abstract}

\section{Introdução}

A modelagem matemática utiliza equações matemáticas com vistas a estudar o comportamento do sistema físico analisado. Um sistema físico pode mudar com o tempo de um estado a outro de tal modo que, sua modelagem pode se tornar uma tarefa difícil, ou até mesmo impraticável. Caso o estado desse sistema em qualquer observação não puder ser predito com certeza, mas se for possível prever a sua probabilidade de ocorrer unicamente a partir do conhecimento do estado do sistema na observação precedente, então, esse processo de mudança (probabilística) de um estado para o outro, é conhecido por cadeia (ou processo) de Markov. Várias são as aplicações das cadeias de Markov, entre as quais estão a Física, que utiliza os sistemas Markovianos em termodinâmica e mecânica e a Ciência da Informação que também utiliza a cadeia de Markov em todo o processamento da informação. A Cadeia de Markov também é bastante utilizada na Economia para estudar diferentes fenômenos, como preços ativos e falhas no mercado [5].

Certamente, um resultado importante nessa teoria, é a convergência dessas cadeias. Recentemente, Silva e Rota [7] analisaram esse problema com modificações em relação ao desenvolvimento descrito por [2] e [4] para calcularem $T^{n} v$ (em vez de $v T^{n}$ ), onde $T$ representa uma matriz quadrada de ordem $r$ de vetores de probabilidade, como fizeram os autores reportados anteriormente.

Entretanto, a demonstração apresentada por [7] mostrou-se, no nosso entendimento, demasiadamente “onerada”. Deve ser observado que o desenvolvimento proposto por [2] foi apresentado de forma concisa, isto é, omitindo passagens de crucial importância no desenvolvimento de sua demonstração. Nesse sentido, é apresentada uma demonstração detalhada do teorema da convergência da cadeia de Markov com as

Palavras-chave: Matriz de transição; Vetor de Estado Estacionário; Convergência.

${ }^{1}$ FFP-UERJ, fabiana_s_b_@hotmail.com; cris_camposs@hotmail.com; lorrene.alc@hotmail.com

2DMAT/FFP-UERJ,jcaraujo_55@yahoo.com.br; rosagmarquez@yahoo.com.br 
alterações necessárias em relação ao desenvolvimento descrito por [2] para ser adaptada ao produto $T^{n} v$. Uma aplicação é proposta e discutida à luz dos diferentes métodos para a obtenção do vetor de estado estacionário.

\section{Método}

Sejam $X_{1}, X_{2}, \ldots, X_{n}, n \in \mathbb{N}$ variáveis aleatórias discretas de probabilidade definidas em um conjunto $E=\left\{e_{1}, \ldots, e_{k}\right\}$, onde $e_{i}, i=1, \ldots, k$ são os estados probabilísticos possíveis de um evento $\varepsilon$. Uma cadeia de Markov é definida pela probabilidade condicional dada por:

$$
P\left(X_{n}=x_{n} \mid X_{n-1}=x_{n-1}\right)=P\left(X_{n}=x_{n} \mid X_{n-1}=x_{n-1}, \ldots, X_{0}=x_{0}\right) .
$$

A probabilidade de o sistema estar no estado $i$ (ou $e_{i}$ ) em qualquer observação, se na observação precedente estava no estado $j$ (ou $e_{j}$ ), é denotada por $P_{i j}$, e é chamada a probabilidade de transição do estado $e_{j}$ ao estado $e_{i}$. A equação (1) indica que tudo que acontece no futuro dependerá do último estado presente e não do passado [6].

A matriz quadrada $P=\left(P_{i j}\right)_{k \times k}$, de ordem $k, \operatorname{com} P_{i j} \geq 0$ e $\sum_{i=1}^{k} P_{i j}=1$, para $j=1, \ldots, k$ é chamada matriz de transição ou matriz estocástica da cadeia de Markov.

A matriz de transição $P$ é regular se existe $n \in \mathbb{N}$, tal que, $P_{i j}^{n}>0$, para todo $i, j=1, \ldots, k$, isto é $P^{n}$ tem todas as entradas estritamente positivas.

\subsection{Formulação analítica}

\section{Proposição}

O produto de matrizes estocásticas é estocástica.

Demonstração: Sejam $A$ e $B$ matrizes estocásticas de ordem $k$. Do produto matricial [1], um elemento genérico $(A B)_{i j}$ desse produto é dado por

$$
(A B)_{i j}=\sum_{r=1}^{k} A_{i r} B_{r j},
$$

onde, $A_{i r}$ e $B_{r j}$ são respectivamente a $i$-ésima linha de $A$ e a $j$-ésima coluna de $B$.

Queremos provar que a soma dos elementos de cada coluna da matriz $A B$ é igual a um, isto é, $\sum_{i=1}^{k}(A B)_{i j}=1$.

$$
\begin{aligned}
& \sum_{i=1}^{k}(A B)_{i j}=\sum_{i=1}^{k}\left(\sum_{r=1}^{k} A_{i r} B_{r j}\right) \\
& =\sum_{r=1}^{k}\left(\sum_{i=1}^{k} A_{i r} B_{r j}\right) \\
& =\sum_{r=1}^{k}\left(\sum_{i=1}^{k} A_{i r}\right) B_{r j} \\
& =\sum_{r=1}^{k}(1) . B_{r j}=1 .
\end{aligned}
$$


Essa proposição, aparentemente ingênua, mostra que se $P$ é regular, $P^{n}$ é também estocástica para algum $n$ natural. De fato, sendo $P$ uma matriz de transição regular, $P$ é uma matriz estocástica e existe $n$ natural, tal que $P_{i j}^{n}>0$, para todo $i, j=1, \ldots, k$. Daí, e da proposição anterior, resulta que a matriz $P_{i j}^{n}$ é estocástica.

O próximo Teorema nos permitirá demonstrar o Teorema de Convergência de Matrizes Regulares (tais teoremas foram adaptados para o produto $P x$ em vez de $x P$, como desenvolvido por [2]).

\section{Teorema 1}

Seja $P_{r \times r}$ uma matriz de transição de ordem $r$ não tendo entradas nulas. Sejam $\varepsilon=\underset{i, j=1, \ldots, r}{\min }\left(P_{i j}\right)$ e $x$ um vetor linha com $m_{o}=\min _{i=1, \ldots, r}\left(x_{i}\right)$ e $M_{o}=\max _{i=1, \ldots, r}\left(x_{i}\right)$. Analogamente, sejam $m_{1}=\min _{j=1, \ldots, r}(x P)$ e $M_{1}=\underset{j=1, \ldots, r}{\max }(x P)$. Então,

$$
\begin{aligned}
& M_{1} \leq M_{0}, \quad m_{1} \geq m_{0}, \\
& M_{1}-m_{1} \leq(1-2 \varepsilon)\left(M_{0}-m_{0}\right) .
\end{aligned}
$$

Demonstração: Seja $x^{\prime}$ o vetor linha obtido de $x$ onde todas as suas componentes foram substituídas por $M_{0}$, exceto uma que continuou com $m_{0}$.

Sem perda de generalidade, podemos supor que o $m_{0}$ ocupe a primeira coordenada de $x^{\prime}$ e que $P_{1 j}=a$ . Assim, a $j$-ésima componente de $x^{\prime} P$, é dada pelo produto do vetor $x^{\prime}$ com a $j$-ésima coluna da matriz $P$, indicada por $P(., j)$.

$$
\left(x^{\prime} P\right)_{j}=x^{\prime} P(., j)=a m_{0}+\sum_{i=2}^{r} M_{0} P_{i j}=a m_{0}+M_{0} \sum_{i=2}^{r} P_{i j} .
$$

Como $\sum_{j=1}^{r} P_{i j}=1$, tem-se que $\sum_{j=2}^{r} P_{i j}=1-a$.

Daí,

$$
\left(x^{\prime} P\right)_{j}=a m_{0}+M_{0}(1-a)=M_{0}-a\left(M_{0}-m_{0}\right) .
$$

Desde que $a \geq \varepsilon$, pois $a \in P$, obtemos:

$$
-a\left(M_{0}-m_{0}\right) \leq-\varepsilon\left(M_{0}-m_{0}\right)
$$

ou

$$
\left(x^{\prime} P\right)_{j} \leq M_{0}-\varepsilon\left(M_{0}-m_{0}\right)
$$

Em particular, como $M_{1}$ é a maior das coordenadas do vetor $x^{\prime} P$ satisfaz a desigualdade

$$
M_{1} \leq M_{0}-\varepsilon\left(M_{0}-m_{0}\right)
$$

Como, $\varepsilon\left(M_{0}-m_{0}\right)>0$, temos que

$$
M_{1} \leq M_{0}
$$

Se o vetor linha $x$ for multiplicado por - 1, a desigualdade (9) fica na forma:

$$
-m_{1} \leq-m_{0}-\varepsilon\left(M_{0}-m_{0}\right)
$$


Para verificar a desigualdade (11), basta usar a desigualdade (9) observando que:

$$
\begin{aligned}
& \max _{i=1, \ldots, r}\left(-x^{\prime}\right)=-m_{o} \quad\left(=" M_{o}^{\prime \prime}\right) \\
& \max _{j=1, \ldots, r}\left(-x^{\prime} P\right)=-m_{1} \quad\left(=" M_{1}^{\prime \prime}\right) \\
& \min _{i=1, \ldots, r}\left(-x^{\prime}\right)=-M_{o} \quad\left(=" m_{o}^{\prime \prime}\right) \\
& \min _{j=1, \ldots, r}\left(-x^{\prime} P\right)=-M_{1} \quad\left(=" m_{1}^{\prime \prime}\right) .
\end{aligned}
$$

Da desigualdade (11) obtemos

$$
m_{1} \geq m_{0}
$$

Adicionando $-m_{1}$ às desigualdades (9) e (10), temos:

$$
M_{1}-m_{1} \leq M_{0}-\varepsilon\left(M_{0}-m_{0}\right)-m_{0}-\varepsilon\left(-m_{0}+M_{0}\right)
$$

ou

$$
M_{1}-m_{1} \leq(1-2 \varepsilon)\left(M_{0}-m_{0}\right) .
$$

Além disso, como $M_{0} \geq M_{1}$ e $m_{0} \leq m_{1}$, tem-se

$$
M_{1}-m_{1} \leq M_{0}-m_{1} \leq M_{0}-m_{0}
$$

Desse modo,

$$
M_{0}-m_{0} \geq M_{0}-m_{1}
$$

Da desigualdade (14) resulta:

$$
\frac{M_{1}-m_{1}}{M_{0}-m_{0}} \leq 1
$$

Da desigualdade (13) tem-se $1-2 \varepsilon \geq 0$ e como $\varepsilon>0$ segue de (16) que

$$
0<\frac{M_{1}-m_{1}}{M_{0}-m_{0}} \leq 1-2 \varepsilon<1
$$

Logo,

$$
0<1-2 \varepsilon<1
$$

O Teorema 1 é uma extensão do lema 4 de Silva e Rota [7] no sentido dos resultados adicionais (10), (12) e (17) obtidos. Também a demonstração aqui apresentada para a desigualdade (13) possui diferenças significativas em relação à realizada pelos referidos autores. Por exemplo, a igualdade (6) não foi demonstrada claramente em [2] e [7] e, a desigualdade (11) foi provada usando uma metodologia diferente da adotada por [7]. Além disso, a desigualdade (17) foi realizada de forma direta em [7], quando no nosso entendimento, haveria de se exigir que $r \geq 2$. 


\section{Teorema 2}

Se $P$ é uma matriz de transição regular, então $P^{n} \rightarrow A$, sendo $A$ uma matriz de probabilidade de ordem $r$, onde cada coluna é formada pelo mesmo vetor de probabilidade $a=\left(a_{1}, a_{2}, \ldots, a_{r}\right)^{T}$.

Demonstração: Suponhamos primeiramente que $P_{i j} \neq 0$, para todo $i, j=1, \ldots, r$ e que $\varepsilon=\min \left(P_{i . j}\right)$. Seja $e_{j}=(0,0, \ldots, 0,1,0, \ldots, 0)$, com a unidade na $j$-ésima posição. Seja $M_{n}=\max _{j=1, \ldots, r} e_{j} P^{n} \quad \mathrm{e}$ $m_{n}=\min _{j=1, \ldots, r} e_{j} P^{n}, n \geq 1$. Isto é, $M_{n}$ e $m_{n}$ são respectivamente a máxima e a mínima componentes do vetor linha $e_{j} P^{n}=P^{n}(j,$.$) , onde e_{j} P^{n}=\left(e_{j} P^{n-1}\right) P$.

Dos resultados (3) e (4) do teorema anterior, tem-se a cadeia não crescente $M_{1} \geq M_{2} \geq \cdots$ e não decrescente $m_{1} \leq m_{2} \leq \ldots$ de modo que usando (13) no estágio $n$ tem-se

$$
M_{n}-m_{n} \leq(1-2 \varepsilon)\left(M_{n-1}-m_{n-1}\right)
$$

ou

$$
M_{n}-m_{n} \leq(1-2 \varepsilon)^{n}\left(M_{0}-m_{0}\right)
$$

Seja $d_{n}=M_{n}-m_{n}, n \geq 1$. Como $0 \leq d_{0} \leq 1$ tem-se

$$
(1-2 \varepsilon)^{n} d_{0} \leq(1-2 \varepsilon)^{n}
$$

Daí, e de (18) resulta:

$$
\begin{aligned}
& 0 \leq d_{n} \leq(1-2 \varepsilon)^{n} d_{0} \leq(1-2 \varepsilon)^{n} . \\
& d_{n} \rightarrow 0 \text { quando } n \rightarrow \infty, \log 0 M_{n} \rightarrow m_{n}
\end{aligned}
$$

Assim, a máxima componente do vetor linha, $e_{j} P^{n}$ é igual à mínima componente deste mesmo vetor linha, digamos que $a_{j}$ seja esse valor em comum, logo a linha $e_{j} P^{n}=\left(a_{j} \ldots a_{j}\right)$.

Repetindo sucessivas vezes o cálculo, $e_{j} P^{n}, j=i, \ldots, r$, tem-se

$$
e_{1} P^{n}=\left(a_{1}, \ldots, a_{1}\right), e_{2} P^{n}=\left(a_{2}, \ldots, a_{2}\right), \ldots, e_{r} P^{n}=\left(a_{r}, \ldots, a_{r}\right)
$$

ou

$$
P^{n}=A=\left[\begin{array}{ccc}
a_{1} & \ldots & a_{1} \\
a_{2} & \ldots & a_{2} \\
\ldots & \\
a_{r} & \ldots & a_{r}
\end{array}\right]
$$

isto é, $P^{n}$ tende a uma matriz $A$ cujas colunas são todas iguais ao vetor $a=\left(a_{1}, a_{2}, \ldots, a_{r}\right)^{T}$. Como $a_{j}$ é o valor comum através da aproximação entre $m_{n}$ e $M_{n}$, é claro que

$$
m_{n} \leq a_{j} \leq M_{n} \text {, para todo } n \geq 1 \text {. }
$$

Em particular,

$$
0<m_{1} \leq a_{j} \leq M_{1}<1 .
$$


Então cada componente $a_{j}$ é positiva. Como $P^{n}$ é estocástica, $\sum_{j=1}^{r} a_{j}=1$.

Caso $P$ seja regular, existe $N \in \mathbb{N}$, tal que $P_{i j}^{N}>0$, daí e usando a prova realizada para o caso do Teorema 1 , em que $P_{i j} \neq 0$, tem-se uma sequência não crescente $0 \leq d_{k N} \leq\left(1-2 \varepsilon^{\prime}\right)^{k}$ onde $d_{k N} \rightarrow 0$, $k \rightarrow \infty$ e $P^{n} \rightarrow A, n \rightarrow \infty$, onde cada coluna de $A$ é um mesmo vetor de probabilidade.

O Teorema 2, é equivalente ao Teorema 5, itens (i, ii) de Silva e Rota [7]. Entretanto, a prova dos referidos autores envolveu o lema 4 e também o lema 3 não utilizado nesse estudo. Além disso, a metodologia adotada fez uso de limite de sequências de vetores, enquanto a descrita nesse artigo baseou-se tão somente nas desigualdades (3), (4) e em (13), todas decorrentes do Teorema 1. Nesse sentido, acreditamos que nossa abordagem ficou mais detalhada quando comparada com [2] e [7].

O próximo teorema mostrará que $P^{n} x_{0}$ se aproxima do vetor estacionário $a$ independente do vetor de probabilidade inicial $x_{0}$, e que $a$ é único no sentido de satisfazer a equação, $P^{n} a=a$. Além disso, a matriz $P$ comuta $\operatorname{com} A$.

\section{Teorema 3}

Se $P$ é uma matriz de transição regular e $A$ e $a$ são dados como no teorema 2, então:

(a) Para qualquer vetor de probabilidade $x_{0}, P^{n} x_{0}$ tende a $a$ quando $n$ tende ao infinito.

(b) $\mathrm{O}$ vetor $a$ é o único vetor de probabilidade tal que $P a=a$.

(c) $P A=A P$.

Demonstração: Como P é regular, pelo teorema 2, $P^{n} \rightarrow A$ quando $n \rightarrow \infty$.

$$
P^{n} x_{0}=A x_{0}=\left(a_{1} \sum_{i=1}^{r} x_{i}, a_{2} \sum_{i=1}^{r} x_{i}, \ldots, a_{r} \sum_{i=1}^{r} x_{i}\right)^{T},
$$

onde, $x_{0}=\left(x_{1}, x_{2}, \ldots, x_{r}\right)^{T}$. Como $x_{0}$ é um vetor de probabilidade, $\sum_{i=1}^{r} x_{i}=1$. Daí, $P^{n} x_{0}=A x_{0}=a$.

Seja $\beta$ um vetor de probabilidade tal que, $P \beta=\beta$, então usando o item (a), $P^{n} \beta=a$ quando $n$ tende a infinito. Por outro lado, $P^{2} \beta=P(P \beta)=\beta, \ldots, P^{n} \beta=\beta$. Logo, $\beta=a$.

$P^{n+1}=P^{n} P=A P$, quando $n$ tende ao infinito. Mas $P A=P P^{n}$, logo $A P=P A$.

O Teorema 3 é equivalente ao Teorema 5, itens (iii e iv) de Silva e Rota [7], mas a prova que fizemos para o item (b) que corresponde ao item (iv) de [7] não fez uso do teorema da unicidade do limite.

\subsection{Aplicação}

A seguir veremos uma aplicação da cadeia de Markov baseada no problema proposto por [1] e aqui ampliada, utilizando três diferentes métodos para a obtenção do vetor estacionário.

Um guarda de transito é designado para controlar o trafego nos oito cruzamentos indicados na Figura 1. Ele é instruído a permanecer em cada cruzamento por uma hora e, em seguida, ou permanecer no mesmo cruzamento ou seguir para um cruzamento adjacente. 
Figura 1. Diagrama do cruzamento de ruas e posição inicial do guarda.

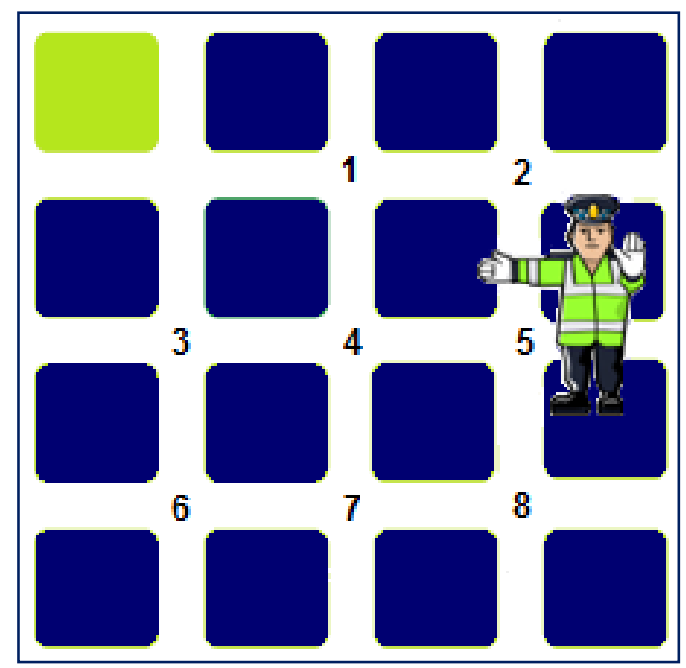

Fonte: Os autores (2017).

Para evitar que ele estabeleça um padrão, ele deve escolher o novo cruzamento de maneira aleatória, com qualquer escolha igualmente provável. Por exemplo, se ele está no cruzamento 5, seu próximo cruzamento pode ser 2, 4, 5 ou 8 , cada um com igual probabilidade $1 / 4$. Cada dia ele recomeça no cruzamento em que parou no dia anterior.

A matriz de transição desta cadeia de Markov com o guarda iniciando seu trabalho na posição 5 é dada abaixo por:

$$
\begin{aligned}
& \text { Cruzamento Velho }
\end{aligned}
$$

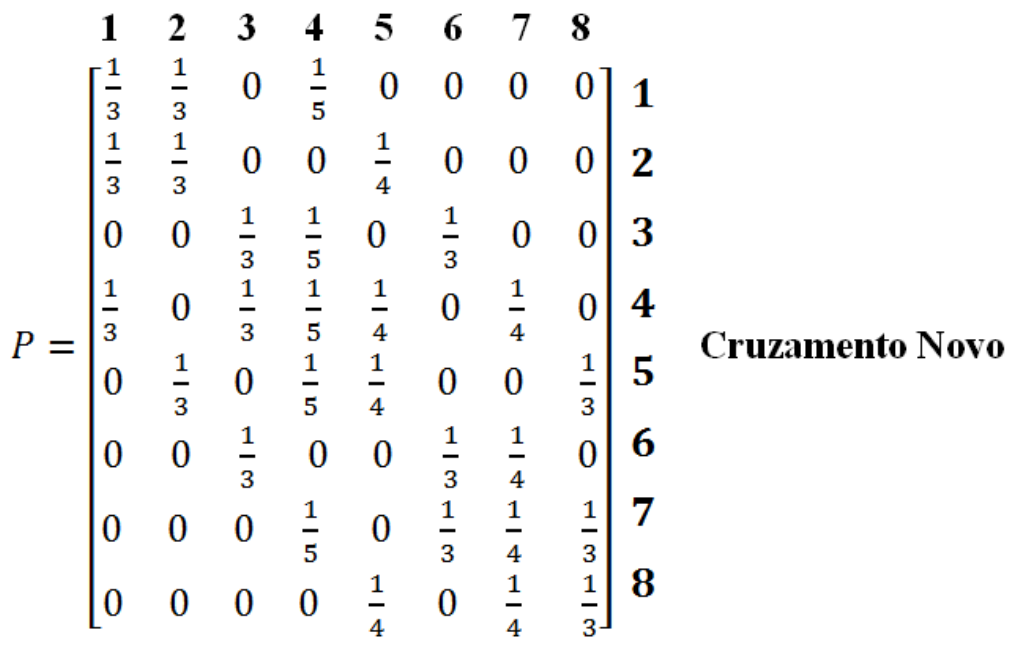

Podemos representar as probabilidades de transição utilizando a representação por grafo, que para este fim é denominado como Diagrama de Transição de Estado. Neste os sentidos das flechas indicam a probabilidade de transição de um estado $i$ para um estado $j$. Para a matriz de transição $P$ dada acima o diagrama fica da seguinte forma conforme pode ser visto na Figura 2. 
Figura 2. Diagrama de transição de estado da matriz $\boldsymbol{P}$. Nota a posição 5 pintada de azul indicado a posição inicial.

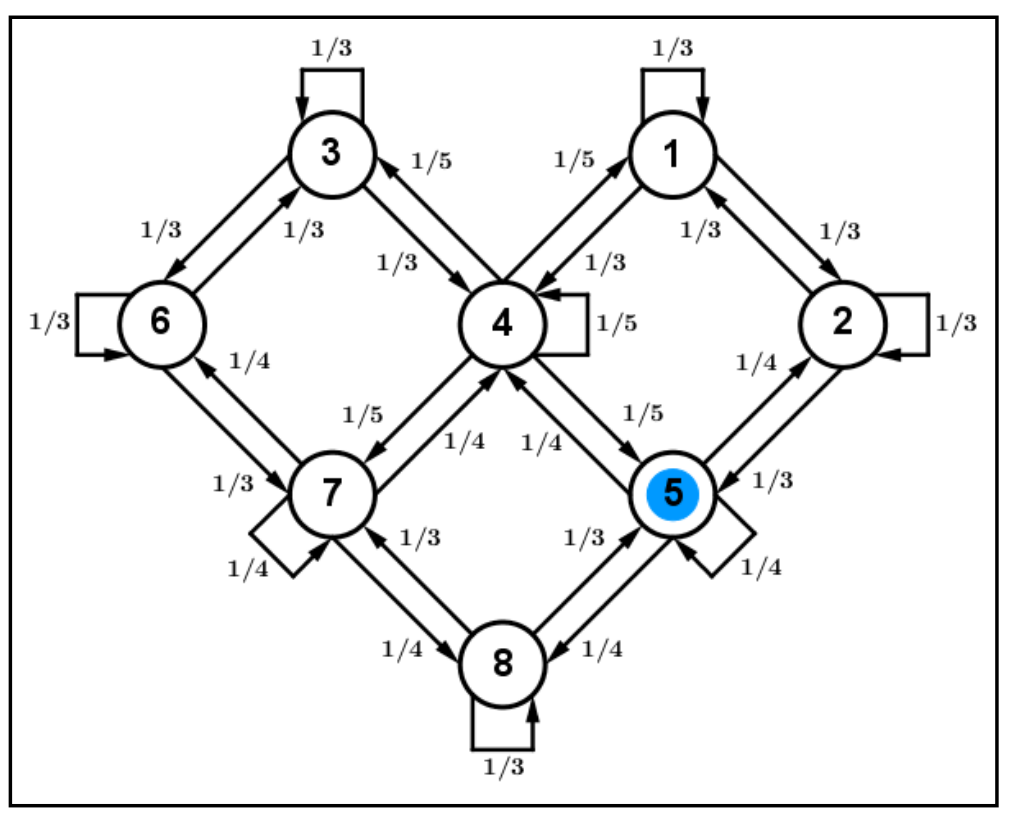

Fonte: Os autores (2017).

Primeiro Método: Utilizando a equação matricial $x^{(n+1)}=P x^{(n)}$ decorrente do Teorema 3 item (a). Supondo que o guarda inicialmente começa no cruzamento 5, suas prováveis localizações, hora à hora, são dadas pelos vetores representados pelas colunas da Tabela 1.

Para todos os valores de $n$ maiores do que 18 , todos os vetores-estado são iguais a $x^{(18)}$ até três casas decimais. Assim, os vetores-estado convergem a um vetor fixo à medida que $n$ cresce. Esse vetor fixo é o vetor estacionário

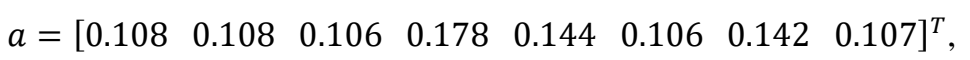

que corresponde ao vetor probabilidade.

Tabela 1. Vetores resultantes desde $x^{(0)}$, indicando a posição inicial 5 , até o vetor o vetor estacionário $x^{(18)}$, indicando a convergência a partir dele.

\begin{tabular}{|c|c|c|c|c|c|c|c|c|c|c|}
\hline \multirow{2}{*}{ Vetor } & \multicolumn{10}{|c|}{ Iteração (n) } \\
\cline { 2 - 10 } & 0 & 1 & 2 & 3 & 4 & 5 & 10 & 15 & 18 & 19 \\
\hline$x_{1}^{(n)}$ & 0 & 0.000 & 0.133 & 0.115 & 0.130 & 0.122 & 0.113 & 0.109 & 0.108 & 0.108 \\
\hline$x_{2}^{(n)}$ & 0 & 0.250 & 0.146 & 0.163 & 0.140 & 0.138 & 0.115 & 0.110 & 0.108 & 0.108 \\
\hline$x_{3}^{(n)}$ & 0 & 0.000 & 0.050 & 0.0391 & 0.0668 & 0.0732 & 0.100 & 0.106 & 0.106 & 0.106 \\
\hline$x_{4}^{(n)}$ & 0 & 0.250 & 0.112 & 0.186 & 0.162 & 0.178 & 0.178 & 0.179 & 0.178 & 0.178 \\
\hline$x_{5}^{(n)}$ & 1 & 0.250 & 0.279 & 0.190 & 0.190 & 0.168 & 0.149 & 0.145 & 0.144 & 0.144 \\
\hline$x_{6}^{(n)}$ & 0 & 0.000 & 0.000 & 0.0499 & 0.0556 & 0.0733 & 0.0988 & 0.105 & 0.106 & 0.106 \\
\hline$x_{7}^{(n)}$ & 0 & 0.000 & 0.133 & 0.104 & 0.130 & 0.125 & 0.138 & 0.142 & 0.142 & 0.142 \\
\hline$x_{8}^{(n)}$ & 0 & 0.250 & 0.146 & 0.152 & 0.124 & 0.121 & 0.108 & 0.107 & 0.107 & 0.107 \\
\hline
\end{tabular}


Segundo Método: Usando a equação $P^{(n)} \rightarrow A$, decorrente do Teorema 2, obtivemos as matrizes abaixo. Para todos os valores de $n$ maiores do que 19 , todos os vetores-estado são iguais a $P^{(19)}$ até três casas decimais. Assim, os vetores-estado de cada coluna da matriz convergem a um vetor fixo à medida que $n$ cresce, resultando numa matriz estacionária $w$. Esse vetor fixo é o vetor estacionário

$$
a=\left[\begin{array}{llllllll}
0.107 & 0.108 & 0.107 & 0.179 & 0.143 & 0.107 & 0.143 & 0.107
\end{array}\right]^{T},
$$

que corresponde ao vetor probabilidade. Note que neste caso não foi necessário uma posição inicial $x_{0}$.

$$
\begin{aligned}
P^{2} & =\left[\begin{array}{llllllll}
0.289 & 0.222 & 0.066 & 0.107 & 0.133 & 0.000 & 0.050 & 0.000 \\
0.222 & 0.306 & 0.000 & 0.117 & 0.146 & 0.000 & 0.000 & 0.083 \\
0.066 & 0.000 & 0.289 & 0.107 & 0.050 & 0.222 & 0.133 & 0.000 \\
0.178 & 0.194 & 0.178 & 0.273 & 0.112 & 0.194 & 0.112 & 0.167 \\
0.178 & 0.194 & 0.066 & 0.090 & 0.279 & 0.000 & 0.133 & 0.194 \\
0.000 & 0.000 & 0.222 & 0.117 & 0.000 & 0.306 & 0.146 & 0.083 \\
0.066 & 0.000 & 0.178 & 0.090 & 0.133 & 0.194 & 0.279 & 0.194 \\
0.000 & 0.083 & 0.000 & 0.100 & 0.146 & 0.083 & 0.146 & 0.278
\end{array}\right] \\
P^{19} & =\left[\begin{array}{llllllllll}
0.108 & 0.108 & 0.106 & 0.107 & 0.108 & 0.106 & 0.107 & 0.107 \\
0.109 & 0.109 & 0.107 & 0.108 & 0.108 & 0.107 & 0.108 & 0.108 \\
0.106 & 0.106 & 0.108 & 0.107 & 0.107 & 0.108 & 0.108 & 0.107 \\
0.180 & 0.179 & 0.179 & 0.180 & 0.178 & 0.178 & 0.178 & 0.178 \\
0.143 & 0.144 & 0.142 & 0.142 & 0.144 & 0.142 & 0.142 & 0.143 \\
0.106 & 0.106 & 0.108 & 0.107 & 0.107 & 0.108 & 0.108 & 0.107 \\
0.142 & 0.142 & 0.144 & 0.143 & 0.143 & 0.144 & 0.144 & 0.143 \\
0.107 & 0.107 & 0.107 & 0.107 & 0.108 & 0.107 & 0.108 & 0.108
\end{array}\right] \\
P^{20} & =\left[\begin{array}{lllllllll}
0.108 & 0.108 & 0.106 & 0.107 & 0.108 & 0.106 & 0.107 & 0.107 \\
0.109 & 0.109 & 0.107 & 0.108 & 0.108 & 0.107 & 0.108 & 0.108 \\
0.106 & 0.106 & 0.108 & 0.107 & 0.107 & 0.108 & 0.108 & 0.107 \\
0.180 & 0.179 & 0.179 & 0.180 & 0.178 & 0.178 & 0.178 & 0.178 \\
0.143 & 0.144 & 0.142 & 0.142 & 0.144 & 0.142 & 0.142 & 0.143 \\
0.106 & 0.106 & 0.108 & 0.107 & 0.107 & 0.108 & 0.108 & 0.107 \\
0.142 & 0.142 & 0.144 & 0.143 & 0.143 & 0.144 & 0.144 & 0.143 \\
0.107 & 0.107 & 0.107 & 0.107 & 0.108 & 0.107 & 0.108 & 0.108
\end{array}\right]
\end{aligned}
$$

Terceiro Método: Usando o item (b) do Teorema 3, tem-se a equação $P w=w$, que é equivalente a resolver o sistema linear $(P-I) w=0$. 


$$
P-I=\left[\begin{array}{cccccccc}
\frac{-2}{3} & \frac{1}{3} & 0 & \frac{1}{5} & 0 & 0 & 0 & 0 \\
\frac{1}{3} & \frac{-2}{3} & 0 & 0 & \frac{1}{4} & 0 & 0 & 0 \\
0 & 0 & \frac{-2}{3} & \frac{1}{5} & 0 & \frac{1}{3} & 0 & 0 \\
\frac{1}{3} & 0 & \frac{1}{3} & \frac{-4}{5} & \frac{1}{4} & 0 & \frac{1}{4} & 0 \\
0 & \frac{1}{3} & 0 & \frac{1}{5} & \frac{-3}{4} & 0 & 0 & \frac{1}{3} \\
0 & 0 & \frac{1}{3} & 0 & 0 & \frac{-2}{3} & \frac{1}{4} & 0 \\
0 & 0 & 0 & \frac{1}{5} & 0 & \frac{1}{3} & \frac{-3}{4} & \frac{1}{3} \\
0 & 0 & 0 & 0 & \frac{1}{4} & 0 & \frac{1}{4} & \frac{-2}{3}
\end{array}\right]
$$

Resolvendo $(P-I) w=0$, chegamos à matriz ampliada usando o método da eliminação de Gauss completo [1] na forma

$$
\left[\begin{array}{rrrrrrrrr}
1 & 0 & 0 & 0 & 0 & 0 & 0 & -1 & 0 \\
0 & 1 & 0 & 0 & 0 & 0 & 0 & -1 & 0 \\
0 & 0 & 1 & 0 & 0 & 0 & 0 & -1 & 0 \\
0 & 0 & 0 & 1 & 0 & 0 & 0 & -\frac{5}{3} & 0 \\
0 & 0 & 0 & 0 & 1 & 0 & 0 & -\frac{4}{3} & 0 \\
0 & 0 & 0 & 0 & 0 & 1 & 0 & -1 & 0 \\
0 & 0 & 0 & 0 & 0 & 0 & 1 & -\frac{4}{3} & 0 \\
0 & 0 & 0 & 0 & 0 & 0 & 0 & 0 & 0
\end{array}\right]
$$

onde $x_{1}=x_{8}, x_{2}=x_{8}, x_{3}=x_{8}, x_{4}=\frac{5}{3} x_{8}, x_{5}=\frac{4}{3} x_{8}, x_{6}=x_{8}, x_{7}=\frac{4}{3} x_{8}$ e $x_{8}$ é a variável livre.

Escolhemos $x_{8}=3$ para obtermos uma base simples, sem frações. Assim,

$$
w=\left[\begin{array}{llllllll}
3 & 3 & 3 & 5 & 4 & 3 & 4 & 3
\end{array}\right]^{T} .
$$

Multiplicando o vetor estacionário $w$ pelo escalar $1 / 28$, onde o denominador é obtido somando as coordenas de $w$, obtemos o vetor probabilidade estacionário

$$
a=\left[\begin{array}{llllllll}
0.107 & 0.107 & 0.107 & 0.179 & 0.143 & 0.107 & 0.143 & 0.107
\end{array}\right]^{T} .
$$

O denominador do escalar, 1/28 pode ser obtido somando as coordenadas de $w$.

\section{Resultados e Discussões}

A Tabela 2 mostra o vetor estacionário calculado pelos três procedimentos descritos anteriormente. As pequenas variações podem ser creditadas aos erros de arredondamento e truncamento realizados pela máquina. No terceiro método não é possível observar os estados probabilísticos da transição da cadeia de Markov. Além do mais, devido à facilidade de programação dos métodos 1 e 2 eles devem ser preferíveis devido a sua praticidade em relação ao método 3 de característica mais algébrica. 
Tabela 2. Vetor estacionário resultante de cada método. Em 1 e 2, $n$ representa o número da iteração na qual ocorreu a convergência.

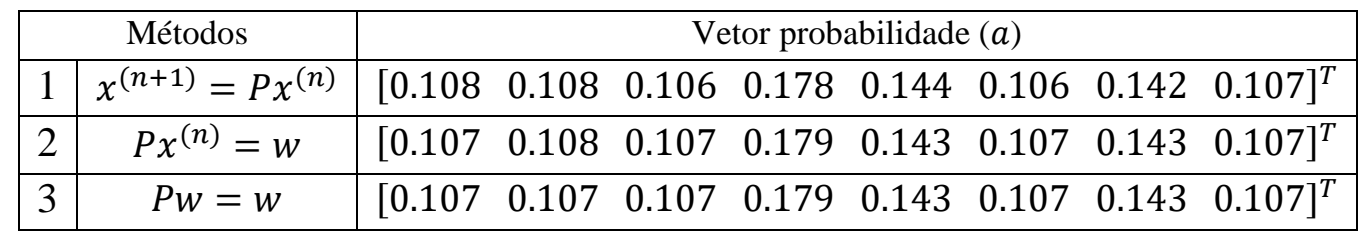

Retomando a análise do problema proposto, as coordenadas desses vetores expõem a probabilidade da posição do guarda após passadas 19 horas, tendo como início a posição 5. Utilizando os resultados do primeiro método, e considerando arredondamentos da máquina, temos a seguinte representação de probabilidade das posições em porcentagem:

Figura 3. Representação dos cruzamentos das ruas com as respectivas probabilidades em porcentagem da posição do guarda de pois de 19 horas.

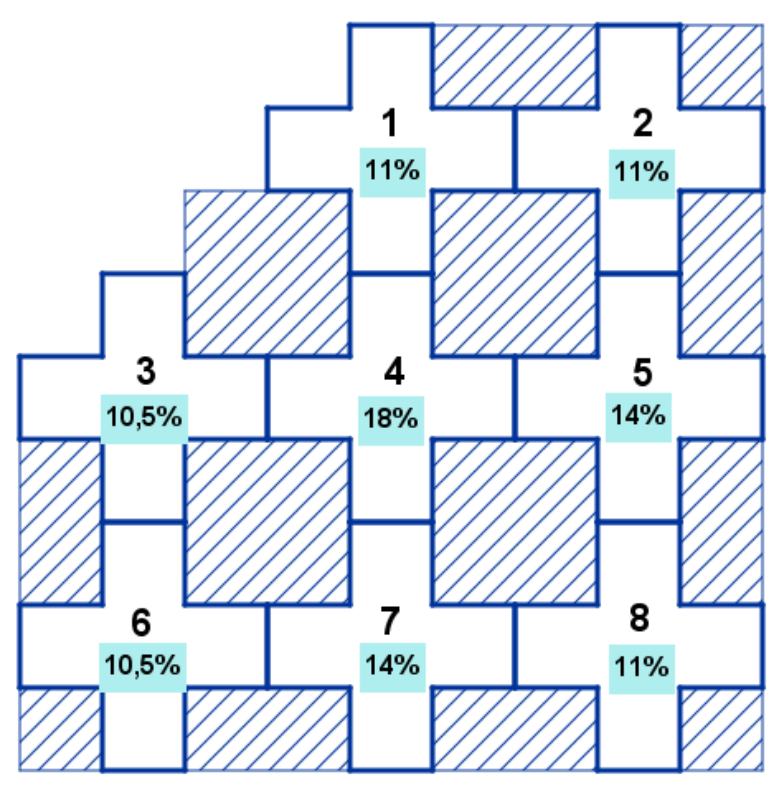

Fonte: Os autores (2017).

Consideramos também uma análise geral utilizando o primeiro método, que nos permite melhor observar os estados probabilísticos da transição da convergência da cadeia de Markov, considerando o guarda em cada um dos outros sete cruzamentos ampliando desse modo, o projeto da aplicação proposto em [1]. Os resultados mostraram pequenas variações no número de interações para alcançar a convergência, porém os vetores estacionários resultante de cada posição inicial mostraram atingir probabilidades concordantes entre eles como mostra a Tabela 3, o que era de se esperar, visto que todos partiram da mesma matriz de transição $P$. Novamente, as pequenas variações nas coordenadas do vetor estacionário pelos diferentes métodos podem ser creditadas aos erros de arredondamento e truncamento realizados pela máquina. 
Tabela 3. Vetores estacionários resultantes utilizando o Método 1 de cada posição inicial do guarda e o número de iterações (horas) necessárias para a convergência.

\begin{tabular}{|c|c|c|c|c|c|c|c|c|c|}
\hline Posição & Número de & \multicolumn{8}{|c|}{ Vetor probabilidade } \\
\hline 1 & 20 & {$[0.109$} & 0.109 & 0.107 & 0.180 & 0.144 & 0.107 & 0.144 & $0.108]^{T}$ \\
\hline 2 & 21 & {$[0.109$} & 0.109 & 0.107 & 0.180 & 0.144 & 0.107 & 0.144 & $0.108]^{T}$ \\
\hline 3 & 19 & {$[0.106$} & 0.106 & 0.108 & 0.178 & 0.141 & 0.108 & 0.142 & $0.106]^{T}$ \\
\hline 4 & 9 & {$[0.107$} & 0.107 & 0.107 & 0.178 & 0.142 & 0.107 & 0.142 & $0.106]^{T}$ \\
\hline 5 & 18 & {$[0.108$} & 0.108 & 0.106 & 0.178 & 0.144 & 0.106 & 0.142 & $0.107]^{T}$ \\
\hline 6 & 21 & {$[0.107$} & 0.107 & 0.109 & 0.180 & 0.144 & 0.109 & 0.144 & $0.108]^{T}$ \\
\hline 7 & 18 & {$[0.106$} & 0.106 & 0.108 & 0.178 & 0.142 & 0.108 & 0.144 & $0.108]^{T}$ \\
\hline 8 & 12 & {$[0.106$} & 0.106 & 0.106 & 0.178 & 0.142 & 0.106 & 0.142 & $0.107]^{T}$ \\
\hline
\end{tabular}

Nosso objetivo foi, portanto, seguir exclusivamente os passos de [2], adaptando-os para o caso $T^{n} v$ de modo que, os principais resultados fossem demonstrados de forma mais compreensível do que a versão sucinta encontrada em [2] e desse modo, pudessem ser acessíveis a um público mais geral. A descrição de três métodos para obter o vetor estacionário de uma cadeia de Markov foi aqui detalhada e utilizada em uma aplicação prática.

\section{Referências}

[1] ANTON, H.; RORRES, C. Álgebra Linear com Aplicações, trad. Claus Ivo Doering. 8. ed. Porto Alegre: Bookman, 2001.

[2] KEMENY, J. G.; SNELL, J. L. Finite Markov chains. New York: Springer-Verlag, 1960.

[3] LAY, D. C. Álgebra Linear e Suas Aplicações. 2. ed. Editora LTC, 1997.

[4] MANOEL, M. R. Cadeias de Markov: uma abordagem voltada para o ensino médio. 2016. $69 \mathrm{f}$. Dissertação (Mestrado Profissional) - Instituto de Matemática, Estatística e Computação Científica, Universidade Estadual de Campinas. Campinas, 2016.

[5] PRASAD, N. R.; ENDER, R. C.; REILY S. T. e NESGOS G. IEEE Conference Publications: Allocation of resources on a minimized cost basis, 1974 IEEE Conference on Decision and including the $13^{\text {th }}$ Symposium on Adaptive Processes.

[6] RUFFINO, P. R. C. Uma iniciação aos sistemas dinâmicos estocásticos. 2. ed. Rio de Janeiro: IMPA, 2009.

[7] SILVA, F. B.; ROTA, I. S. Convergência de matrizes estocásticas regulares. C.Q.D. - Revista Eletrônica Paulista de Matemática, Bauru, v. 8, p. 4-14, dez. 2016. Edição Iniciação Científica. 